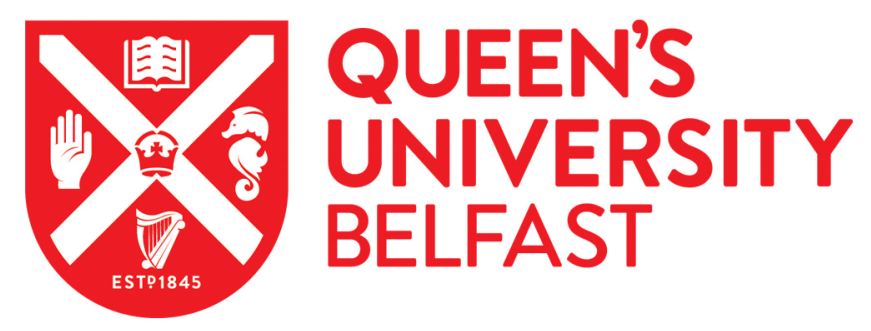

\title{
A Note on Networks of Collaboration in Multimarket Oligopolies
}

Billand, P., Bravard, C., Chakrabarti, S., \& Sarangi, S. (2014). A Note on Networks of Collaboration in Multimarket Oligopolies. Annals of Regional Science, 53(2), 325-335. https://doi.org/10.1007/s00168-013-0577-x

\author{
Published in: \\ Annals of Regional Science
}

Document Version:

Peer reviewed version

Queen's University Belfast - Research Portal:

Link to publication record in Queen's University Belfast Research Portal

\section{Publisher rights}

Copyright 2013 Springer-Verlag Berlin Heidelberg.

This work is made available online in accordance with the publisher's policies. Please refer to any applicable terms of use of the publisher.

\section{General rights}

Copyright for the publications made accessible via the Queen's University Belfast Research Portal is retained by the author(s) and / or other copyright owners and it is a condition of accessing these publications that users recognise and abide by the legal requirements associated with these rights.

Take down policy

The Research Portal is Queen's institutional repository that provides access to Queen's research output. Every effort has been made to ensure that content in the Research Portal does not infringe any person's rights, or applicable UK laws. If you discover content in the Research Portal that you believe breaches copyright or violates any law, please contact openaccess@qub.ac.uk. 


\title{
A Note on Networks of Collaboration in Multi-market Oligopolies
}

\author{
Pascal Billand $^{a}$, Christophe Bravard ${ }^{b}$, Subhadip Chakrabarti ${ }^{c}$ Sudipta Sarangi $^{d}$
}

\begin{abstract}
In this note, we extend the Goyal and Joshi's model of network of collaboration in oligopoly to multi-market situations. We examine the incentive of firms to form links and the architectures of the resulting equilibrium networks in this setting. We also present some results on efficient networks.
\end{abstract}

JEL code: C70, L13, L20, D85

Key Words: R\&D Collaborations, Network Formation, Multi-market Oligopolies.

${ }^{a}$ Université de Lyon, F-69003, France, CNRS Gate Lyon Saint-Etienne.

email: pascal.billand@univ-st-etienne.fr, pascal.billand@univ-st-etienne.fr

${ }^{b}$ Université Grenoble 2, UMR 1215 GAEL F3800 Grenoble, France, CNRS, Gate Lyon Saint-Etienne, France.

email: pascal.billand@univ-st-etienne.fr, christophe.bravard@univ-st-etienne.fr

${ }^{c}$ School of Management and Economics, Queen's University Belfast, Northern Ireland, United Kingdom

email:s.chakrabarti@qub.ac.uk

${ }^{d}$ Department of Economics, Virginia Tech and Louisiana State University. email: sarangi@lsu.edu 


\section{Introduction}

Research collaborations among firms take a variety of forms, such as joint ventures, technology sharing or joint R\&D (Hagedoorn, 2006). These collaborations are often bilateral and non exclusive, and form a network of collaborative links between firms. By forming collaborative links, firms alter the competitive positions of competitors and influence market structure and performance. It is then important to develop models of network formation between firms in order to predict the architectures of the networks that should emerge and examine to what extent these networks are socially efficient.

Goyal and Joshi (GJ, 2003) propose a model of network formation which addresses these questions for situations in which firms compete on a oligopoly market. Collaborative links between firms lower marginal costs. However these links are costly and the costs are fixed and uniform across links. Westbrock (2010) investigates efficient networks in this setting albeit with differentiated products. Certain architectures emerge as candidates for stable and efficient networks. In the case that has maximum relevance

for us, namely quantity competition in a homogeneous oligopoly with small costs of link formation, the complete network is both stable and efficient. In more general settings, stars, interlinked stars and dominant group architectures emerges as candidates for stable and efficient networks.

Now competition between firms in few markets is a prominent feature of modern economies. In a seminal paper, Bulow et al. (1985) show how the introduction of multimarket competition can produce quite unexpected findings compare to standard results in industrial organization. We can then ask what happens to equilibrium and efficient networks when we introduce multimarket competition in the GJ's model of formation of collaborative networks. 
This paper gives a first insight at this question. We assume for purposes of simplicity heterogeneous product markets, with linear demand curves and quadratic cost functions, where multimarket competitors can form quality products enhancing collaborative links on one market. ${ }^{1}$ Our paper is different from the two earlier papers that follows the framework introduced by Bloch (1995) in the sense that links reduce marginal costs. We follow the line of reasoning laid down by Chakrabarti and Haller (2011). In that paper, firm can make costly investments that increase its demand and reduce the demand of other firms. These investments are interpreted as negative advertising. Here, investments increase the demand of both firms making the investment and interpreted as collaborative $R \& D$ activity. These investments take the form of costly links where the link increases the demand of both firms forming the link by a fixed amount. We restrict ourselves to quantity competition and use a similar demand function to that of Singh and Vives (1984), Westbrock (2010) and Chakrabarti and Haller (2011).

Our findings concerning equilibrium networks are quite similar to those of GJ (2003), that is equilibrium networks have still a dominant group architecture, i.e. a group of firms are linked to each other while other firms have no links at all. We show that this result follows from the fact that our collaborative multimarket game qualifies as playing the field games (Goyal and Joshi, 2006), with payoff function satisfying convexity in own links as well as the strategic substitutes property with regard to quantities. However, using Bulow et al. (1985) analysis, we show that contrary to the model of GJ (2003) there exist situations where competitors do not form collaborative links in order to increase the

\footnotetext{
${ }^{1}$ Considering that firms can form links on one market only is admittedly restrictive. However, it allows us to deal with situations where products innovation is a strategic issue for only one market, and there is little to gain by forming collaborative links that will increase the quality of the product on the second market. Besides this restrictive assumption simplifies the analysis and allows us to give a first insight on the impact of multi-market competition on the collaborative networks.
} 
quality of their products even if these links have no costs. This result follows from the fact that there are joint diseconomies across markets and goods produced by competing firms are strategic substitutes. It illustrates how the intermarket effect play a key role in the incentive of firms to collaborate.

With regard to efficient networks, though we cannot arrive at a precise characterization of these networks, we can derive some interesting properties of such networks and restrict the set of networks that are efficient into a small group. More precisely, we show that efficient networks belong to the class of Nested-Split Graphs. ${ }^{2}$ These architectures have interesting features. In particular, they consist of only one component, that is efficient networks are such that firms which have formed links are all directly or indirectly linked. Moreover the diameter of the non-singleton component is very low since firms are at most at distance 2 from each other. Besides it turns out that equilibrium and efficient networks do not coincide.

The rest of the paper proceeds as follows. Section 2 introduces the model and discusses the notation and terminology. Section 3 examines equilibrium networks, while Sections 4 deals with efficient networks. Section 5 concludes.

\section{Framework}

\subsection{The Multi-market Cournot Model}

Suppose there are $n$ firms indexed by $i=1,2, \ldots, n$ (where $n \geq 3$ ) that compete à la Cournot in two inter-related markets 1 and 2. The set of all firms is denoted by $N$. We denote by $q_{i}$ and $Q_{i}$ the quantities sold by firm $i$ on markets 1 and 2 respectively,

\footnotetext{
${ }^{2}$ We thank one of the referees for pointing out this general characteristic of efficient networks in our framework.
} 
and by $p_{i}$ and $P_{i}$ the prices of firm $i$ 's products on these markets. Focusing on the most interesting case, we assume that competitors' products are strategic substitutes and there are diseconomies of scope across markets. ${ }^{3}$.

More specifically, demand in markets 1 and 2 for firm $i$ are respectively given by

$$
p_{i}=\alpha_{i}-q_{i}-\sum_{j \neq i} q_{j} ; \quad P_{i}=\beta_{i}-Q_{i}-\sum_{j \neq i} Q_{j} .
$$

The cost function of a firm $i$ is given by

$$
C_{i}\left(q_{i}, Q_{i}\right)=\frac{1}{2}\left(q_{i}+Q_{i}\right)^{2}
$$

Since $\frac{\partial^{2} C_{i}\left(q_{i}, Q_{i}\right)}{\partial q_{i} \partial Q_{i}}=2\left(q_{i}+Q_{i}\right)>0$, there are diseconomies of costs across markets.

The profits of each firm $i \in N$ can be written as:

$$
\pi_{i}=\left(\alpha_{i}-q_{i}-\sum_{j \neq i} q_{j}\right) \cdot q_{i}+\left(\beta_{i}-Q_{i}-\sum_{j \neq i} Q_{j}\right) \cdot Q_{i}-\frac{1}{2}\left(q_{i}+Q_{i}\right)^{2} .
$$

\subsection{Quality Improving Links and Collaborative Networks}

Assume that firms can improve the quality of their products via collaborative links in only market 1 . More specifically, we suppose that for each firm $i \in N$ we have

$$
\alpha_{i}(g)=\gamma_{0}+\gamma \cdot \eta_{i}(g)
$$

where $\eta_{i}(\mathrm{~g})$ is the number of links formed by firm $i$ in the network $g$. Link formation is costly and the cost of forming each link is given by $c \geq 0$ and is incurred by both firms forming the link.

\footnotetext{
${ }^{3}$ Formally, let $\pi_{i}\left(q_{1}, \ldots, q_{i}, \ldots q_{n}, Q_{1}, \ldots, Q_{i}, . . Q_{n}\right)$ be the profits function of firm $i$. Competitors' products are strategic substitutes on market 1 whenever $\frac{\partial^{2} \pi_{i}}{\partial q_{i} \partial q_{j}}<0$. Let $C_{i}\left(q_{i}, Q_{i}\right)$ be the cost function of a firm $i$. There are diseconomies (economies) of scope across markets whenever $\frac{\partial^{2} C_{i}\left(q_{i}, Q_{i}\right)}{\partial q_{i} \partial Q_{i}}>(<) 0$
} 
A collaborative network $g$ is a list of pairs of firms who are linked to each other. For simplicity, we denote the link between $i$ and $j$ (where $i \neq j$ ) by $i j$. So $i j \in g$ indicates $i$ and $j$ are linked in the network $g$. A network $g$ is a formal descriptions of the pair-wise collaborative links that exist between firms. Let $\mathcal{G}$ denote the set of all networks. We let $g+i j$ denote the network formed by adding the link $i j$ to the network $g, g-i j$ denote the network formed by deleting the link $i j$ from the network $g$.

A firm $i$ 's neighborhood $N_{i}(g)$ is given by $\{j \in N \backslash\{i\} \mid i j \in g\}$. We define as $L_{i}(g)=$ $\left\{i j \in g \mid j \in N_{i}(g)\right\} \subset g$ the set of links in which firm $i$ is involved. Let $\lambda(g)=\frac{1}{2} \sum_{i \in N} \eta_{i}(g)$ be the total number of links in a network $g$. For any $h \subset g$, let $g-h$ denotes the network formed by deleting the set of links $h$ from the network $g$. Similarly, for $h \subset g^{N} \backslash g, g+h$ denotes the network formed by adding the links set $h$ to the network $g$. Moreover we define $g_{-i}$ as the resulting network once we remove all firm $i$ 's links from the network $g$.

A path in $g$ connecting $i$ and $j$ is a set of distinct firms $\left\{i_{1}, i_{2}, \ldots, i_{p}\right\} \subset N$ with $p \geq 2$ such that $i_{1}=i, i_{p}=j$, and $\left\{i_{1} i_{2}, i_{2} i_{3}, \ldots, i_{p-1} i_{p}\right\} \subset g$. We say $i$ and $j$ are connected to each other if a path exists between them and they are disconnected otherwise. Given a network $g$, we define a component as a set $C(g) \subset N$ such that for all $i, j \in C(g)$ there exists a path between $i$ and $j$ and there does not exist a path between a player $i \in C(g)$ and a player $j \notin C(g)$. A component $C(g)$ is complete if for all distinct $i, j \in C(g)$, $i j \in g$. Firms who are not connected in the network $g$ are named singletons. We define three network architectures that play a role in this paper. The network $g^{N}$ in which all firms are linked with each other is referred to as the complete network and the network $g^{0}$ in which all firms are singletons is referred to as the empty network. The dominant group architecture $g^{k}$ is characterized by one complete non-singleton component with $\left|N^{k}\right| \geq 2$ and $n-\left|N^{k}\right|$ singletons. 


\subsection{Equilibrium and Efficient Networks}

A network is a strongly pair-wise stable network ${ }^{4}$ if

(a) for all $i \in N$ and $h \subset L_{i}(g), \pi_{i}(g) \geq \pi_{i}(g-h)$, and

(b) for all $i \in N, j \in N$, and $i j \notin g$, if $\pi_{i}(g+i j)>\pi_{i}(g)$ then $\pi_{j}(g+i j)<\pi_{j}(g)$.

For any network $g$, and $h \subset g^{N} \backslash g$, we denote the variation of benefit from links formation by $\Delta \pi_{i}(g, h)=\pi_{i}(g+h)-\pi_{i}(g)$.

Next, we define efficient networks. Let $C S$ be consumers' surplus function and $\Pi$ be firms' aggregate profits function. The welfare function $W$ is given by:

$$
W(g)=C S(g)+\Pi(g)
$$

A network $g$ is an efficient network if $g$ maximizes the welfare function, that is $W(g) \geq$ $W\left(g^{\prime}\right)$ for all $g^{\prime} \neq g$. We say that $g$ is efficient for firms (consumers) if it maximizes the aggregate profits of firms (surplus of consumers). For any network $g$, and $h \subset g^{N} \backslash g$, we denote the variation in welfare and total profits resulting from link formation by $\Delta W(g, h)=W(g+h)-W(g)$ and $\Delta \Pi(g, h)=\Pi(g+h)-\Pi(g)$ respectively.

\section{Characterization of Equilibrium Networks}

Although the payoff functions are quite complicated, this game has features similar to the game analyzed by Goyal and Joshi (2006). Hence we will invoke their results here.

Definition 1 A network game is called playing the field game if the payoff function of firm $i$ is a function of her degree, $\eta_{i}(g)$, and the total number of links in $g_{-i}, \lambda\left(g_{-i}\right)$ :

$$
\pi_{i}(g)=\Phi\left(\eta_{i}(g), \lambda\left(g_{-i}\right)\right) .
$$

\footnotetext{
${ }^{4}$ Our definition can be seen as a generalization of stable networks given in GJ (2003), since in GJ no firm has an incentive to delete one of its links or all its links in a stable network.
} 
Definition 2 The payoff function $\Phi$ is convex in its own links if the marginal returns $\Phi(k+1, l)-\Phi(k, l)$ is strictly increasing in $k$.

Definition 3 Suppose $l^{\prime}>l$. The payoff function $\Phi$ satisfies the strategic substitutes property if $\Phi\left(k+1, l^{\prime}\right)-\Phi\left(k, l^{\prime}\right)<\Phi(k+1, l)-\Phi(k, l)$.

It is easy to check from the equilibrium profits function, $\Phi(k, l)$, given in Appendix 6.1. that the collaborative multimarket game qualifies as playing the field game. Furthermore, we show that this function satisfies convexity in own links as well as the strategic substitutes property. Indeed, define

$$
\Delta \Phi(k, l)=\Phi(k+1, l)-\Phi(k, l)
$$

. The equilibrium profits function satisfies convexity in own links, since we have

$$
\Delta \Phi(k+1, l)-\Delta \Phi(k, l)=\frac{\left(11 n^{4}+44 n^{3}-6 n^{2}-100 n+83\right) \gamma^{2}}{9(n+3)^{2}(n+1)^{2}}>0
$$

Likewise, the equilibrium profits function satisfies the strategic substitutes property, since we have

$$
\Delta \Phi(k, l+1)-\Delta \Phi(k, l)=\frac{-2 \gamma^{2}\left(11 n^{3}+62 n^{2}+91 n+4\right)}{\left.(n+3)^{2}(n+1)^{2}\right)}<0 .
$$

Therefore, we get from GJ (2006, p.327, Proposition 3.1) the following corollary.

Corollary 1 The strongly pair-wise stable network is either complete or empty or has a dominant group architecture.

Remark 1. We can check that the empty network can be a strongly pair-wise stable network, even when costs of forming links are null. For instance, suppose $n=6$, $\gamma_{0}=100, \gamma=0.05, \beta_{i}=400$ for all $i \in N$, and $f=0$. We have $\Delta \Phi(0,0)=-0.09<0$, 
so no firm has an incentive to form a link in the empty network, and the latter is a strongly pair-wise stable network. It follows that in some situations firms will not form collaborative links in order to increase the quality of their products even if these links have no costs.

The intuition behind this fact, inspired from Bulow et al. (1985), is as follows: The formation of a link by firms $i$ and $j$ in market 1 enhances the quality of their product in this market and leads these firms to produce more for this market. Due to joint diseconomies across markets this reduces marginal profitability for firms $i$ and $j$ in market 2 and induces these firms to lower their quantities on this market. Because of strategic substitutability, rival firms will increase their quantities on market 2 and this induces a loss in market 2, and possibly an overall loss, for firms $i$ and $j$. Note that in the one-market Cournot model of GJ (2003), the dominant group architecture can emerge only if there are substantial link formation costs.

Remark 2. As in GJ we find that the impact of the costs of link formation on the size of the non-singleton component is non-monotonic: for low costs, the complete network is uniquely stable, for moderate costs only networks with relatively large dominant groups are stable, for high costs, only medium size non-singleton components are stable (small and large groups are not sustainable), while for very high costs, the empty network is uniquely stable. The example below illustrates the fact that for high costs, only medium size non-singleton components are stable.

Example 1 Let $n=50, \gamma_{0}=749, \gamma=0.45, \beta_{i}=400$, for all $i \in N$. Straightforward computations show that for $f=9.7$, only the empty network and dominant-group networks, $g^{k}$, such that $k$ is at least equal to 7 and at most equal to 21 can be strongly pair-wise stable. 
Remark 3. In the case with Economies of scope across markets, there is no tension across the markets anymore. The formation of a link by firms $i$ and $j$ in market 1 enables both firms to produce larger quantities on both markets. Thus profits of both firms increase in equilibrium. Clearly, under zero link costs, the complete network is the only equilibrium network, i.e. we obtain the same result as in Goyal and Joshi model (2003) where firms compete only on one market.

\section{Characterization of Efficient Networks}

Efficient networks are hard to characterize and we provide a partial characterization for $n \geq 4$ firms. ${ }^{5}$.The first lemma present a result on the consumers' surplus function which simplifies the characterization of efficient networks (the proof is in appendix).

Lemma 1 For any network g, (i) the consumers' surplus function only depends on the total number of collaborative links formed by firms, and (ii) this surplus function is convex in the total number of links formed.

Proof The proof of (i) and (ii) is straightforward since the expression of consumers' surplus function is given by

$$
C S(g)=\frac{\left(n^{2}+4 n+5\right)\left(\bar{\alpha}^{2}+\bar{\beta}^{2}\right)-4(n+2) \bar{\alpha} \bar{\beta}}{\left(3+4 n+n^{2}\right)^{2}},
$$

where $\bar{\alpha}=n \gamma_{0}+2 \gamma \lambda(g)>0$, and $\bar{\beta}=\sum_{i=1}^{n} \beta_{i}>0$.

The following lemma considers a network $g$ in which links $i k$ and $i m$ do not exist, while firm $m$ has formed at least as many links as firm $k$ in $g$. This lemma allows us to state that if total profits of firms increase when the link $i k$ is added to the network $g$,

\footnotetext{
${ }^{5}$ Goyal and Joshi (2003) do not provide efficiency results for the one market case stating that this is a hard problem.
} 
then total profits also increases when the link $i m$ is adding to the network $g+i k$ and this increase is of a greater magnitude (the proof of this lemma is in appendix).

Lemma 2 For any network $g$ and firms $i, k, m \in N$ such that $i k, i m \notin g$ and $\eta_{m}(g) \geq$ $\eta_{k}(g)$, we have $\Delta \Pi(g+i k, i m)>\Delta \Pi(g, i k)$

From Lemmas 1 and 2 we obtain the following lemma (the proof is in appendix).

Lemma 3 For any network $g$ and firm $i$ such that $i k, i m \notin g$ and $\eta_{m}(g) \geq \eta_{k}(g)$, we have $\Delta W(g+i k, i m)>\Delta W(g, i k)$.

The proposition that follows from lemma 3 gives an important characteristic of efficient networks.

Proposition 1 Efficient networks g belong to the class of Nested-Split Graphs (NSG).

Proof First we show that in an efficient network $g$, the following condition is satisfied $(C 1)$ : if $i j \in g$ and $i k \notin g$, then $\eta_{j}(g)>\eta_{k}(g)$. Let $\Delta W(g, i j)$ be the marginal change in welfare when the link $i j$ is added to the network $g$. Suppose there exists an efficient network $g$ and $i j \in g$ and $i k \notin g$. Then, $\Delta W(g-i j, i j) \geq 0$. Suppose, towards a contradiction, $\eta_{k}(g) \geq \eta_{j}(g)$. This implies by Lemma 3 that $\Delta W(g, i k)>0$ contradicting that $g$ is efficient. Hence, for all $k$ such that $i k \notin g, \eta_{k}(g)<\eta_{j}(g)$.

Second, we show that if a network $g$ satisfies the condition $(C 1)$, then $g$ belongs to the class of NSG. Belhaj, Bervoets, and Deroïan (2013, p.9) define a NGS as a graph which satisfies the following condition $(C 2)$ : if $i j \in g$ and $\eta_{k}(g) \geq \eta_{j}(g)$, then $i k \in g$.

Conditions $(C 1)$ and $(C 2)$ are clearly equivalent. It follows that if $g$ is an efficient network, then $g$ belongs to the class of NSG. 
There is an interesting effect behind Condition $(C 1)$. Let a network $g$ and firms $i, k, m \in N$ such that $i k \in g$ and $i m \notin g$. Suppose that we substitute the link $i m$ to the link $i k$ in $g$. The total number of links is the same in network $g$ as in network $g-i k+i m$. It follows from Lemma 1 (i) that the consumer surplus does not change when we substitute the link $i m$ to the link $i k$ in the network $g$. Therefore consumers' surplus plays no role in the result of Proposition 1. Moreover, since the total number of links is the same in the network $g$ as in the network $g-i k+i m$, the total costs of forming links also plays no role in the result of Proposition 1. Therefore, the result of Proposition 1 lies only on the total profits (gross of the costs of forming links) of firms. This Proposition highlights the fact that if firms could act in a cooperative way at the links formation stage of the multi-market oligopoly game, then they would have an incentive to act toward some kind of concentration (of increase of the Gini coefficient) of links distribution among them: it would be better for firms (and for social welfare) that a firm poorly linked firm $k$ gives up to a collaboration it has established with a firm $i$ in network $g$ and let firm $i$ set up an additional collaboration with a firm $m$ which has already set up more collaborations that firm $k$ in $g$.

Remark 3. Since efficient networks belong to the class of NGS, it follows that if a network $g$ is an efficient network, then $g$ has two properties (i) $g$ contains at most one component. (ii) The geodesic distance between any two connected firms in $g$ is at most 2. This means that in an efficient network all firms which have formed links are connected, and connected firms are very close one from each other.

Remark 4. It is worth noting that the architectures of strongly pair-wise stable and efficient networks share some similarities. First, in both cases all firms that have formed collaborative links are directly or indirectly linked together. Moreover, in both cases too 
the maximal distance between these firms is very low: one for strongly pair-wise stable networks, two for efficient networks. However as illustrated by the following example, for some parameters values, architectures of strongly pair-wise stable and efficient networks are very different.

Example 2 Let $n=50, \gamma_{0}=749, \gamma=0.45, \beta_{i}=400$, for all $i \in N$. We know from Example 1 that for $f=9.7$, only the empty network and dominant-group networks, $g^{k}$, such that $k$ is at least equal to 7 and at most equal to 21 can be strongly pair-wise stable. Straightforward computations show that these architectures are less efficient than the complete network.

\section{Conclusion}

In this paper, our aim was to ask what happens to equilibrium and efficient networks when we introduce multimarket competition in the GJ's model of formation of collaborative networks. We have shown that although equilibrium architectures are of the same type as in GJ, a complete network needs not materialize in equilibrium when costs of links are null as it is the case in GJ. With regard to efficiency, we have shown that efficient networks belong to the class of NGS. Therefore, efficient networks have the interesting features of this class of graphs. In particular, they consist of only one component, that is efficient networks are such that firms which have formed links are all directly or indirectly linked. Moreover the diameter of the non-singleton component is very low since firms are at most at distance 2 from each other.

We now briefly discuss some issues that could be explored in future work. First, we need to examine the case where firms can form links on both markets. This will allow us to check the robustness of our results and to know in particular whether other types 
of architectures can emerge as strongly pair-wise stable networks. Second, it will be interesting to adapt the model of Goyal and Moraga-Gonzales (2001), and to introduce in our model the possibility for each firm to unilaterally choose a level of effort in R\&D in order to increase the quality of its product, given the collaboration network. This will allow us to us to examine the impact of bilateral $R \& D$ collaboration on the level of R\&D activities.

\section{Appendix}

\subsection{Equilibrium profits in Multi-market equilibrium}

Differentiating (3) with respect to $q_{i}$ and $Q_{i}$ for each firm $i \in N$, we obtain the equilibrium quantities of each firm $i$ :

$$
\begin{aligned}
& q_{i}^{*}=\frac{\left(2 n^{2}+6 n+1\right) \alpha_{i}-\left(n^{2}+3 n-1\right) \beta_{i}-(2 n+5) \sum_{j \neq i} \alpha_{j}+(n+4) \sum_{j \neq i} \beta_{j}}{3\left(3+4 n+n^{2}\right)} ; \\
& Q_{i}^{*}=\frac{\left(2 n^{2}+6 n+1\right) \beta_{i}-\left(n^{2}+3 n-1\right) \alpha_{i}-(2 n+5) \sum_{j \neq i} \beta_{j}+(n+4) \sum_{j \neq i} \alpha_{j}}{3\left(3+4 n+n^{2}\right)}
\end{aligned}
$$

In order to ensure that each firm produces strictly positive quantities in equilibrium, we will assume that the parameters $\gamma_{0}, \gamma,\left\{\beta_{i}\right\}$ are such that for each firm $i \in N$ we have both :

$$
\begin{gathered}
\gamma_{0}>\frac{\gamma(n-1)(n-2)(2 n+5)+\left(n^{2}+3 n-1\right) \beta_{i}-(n+4) \sum_{i \neq j} \beta_{j}}{3(n+2)}, \\
\gamma_{0}>\frac{-\gamma(n-1)\left(n^{2}+3 n-1\right)+\left(2 n^{2}+6 n+1\right) \beta_{i}-(2 n+5) \sum_{i \neq j} \beta_{j}}{3}
\end{gathered}
$$


We obtain the second stage equilibrium profits by replacing $q_{i}$ and $Q_{i}$ by their equilibrium values, $q_{i}^{*}$ and $Q_{i}^{*}$, in (3). Moreover, by replacing in the equilibrium profits $\alpha_{i}$ by $\gamma_{0}-\gamma \eta_{i}$ and $\sum_{j \neq i} \alpha_{j}$ by $n \gamma_{0}+\sum_{j \neq i} \eta_{j}-\eta_{i}{ }^{6}$, the equilibrium profits function is given by $\pi_{i}(g)=\Phi\left(\eta_{i}(g), \lambda\left(g_{-i}\right)\right)$.

\subsection{Proof of Lemma 1, Lemma 2 and Lemma 3}

Proof of Lemma 1. Consider the total consumer surplus of agents in both markets. It is given by

$$
C S=\sum_{i}\left(\alpha_{i}-p_{i}^{*}\right) q_{i}^{*}+\sum_{i}\left(\beta_{i}-P_{i}^{*}\right) Q_{i}^{*}
$$

where $p_{i}^{*}=\alpha_{i}-\sum_{i=1}^{n} q_{i}^{*}$ and $P_{i}^{*}=\beta_{i}-\sum_{i=1}^{n} Q^{*}$ denotes prices in both markets at equilibrium.

Let $\bar{q}=\sum_{i=1}^{n} q_{i}, \bar{Q}=\sum_{i=1}^{n} Q_{i}, \bar{\alpha}=\sum_{i=1}^{n} \alpha_{i}$, and $\bar{\beta}=\sum_{i=1}^{n} \beta_{i}$. We have,

$$
\begin{aligned}
C S & =\sum_{i}\left(\alpha_{i}-p_{i}^{*}\right) q_{i}^{*}+\sum_{i}\left(\beta_{i}-P_{i}^{*}\right) Q_{i}^{*} \\
& =\sum_{i}\left(\bar{q}^{*}\right) q_{i}^{*}+\sum_{i}\left(\bar{Q}^{*}\right) Q_{i}^{*} \\
& =\left(\bar{q}^{*}\right)^{2}+\left(\bar{Q}^{*}\right)^{2} \\
& =\left[\frac{(n+2) \bar{\alpha}-\bar{\beta}}{3+4 n+n^{2}}\right]^{2}+\left[\frac{(n+2) \bar{\beta}-\bar{\alpha}}{3+4 n+n^{2}}\right]^{2} \\
& =\frac{\left(n^{2}+4 n+5\right)\left(\bar{\alpha}^{2}+\bar{\beta}^{2}\right)-4(n+2) \bar{\alpha} \bar{\beta}}{\left(3+4 n+n^{2}\right)^{2}} .
\end{aligned}
$$

The proof of $(i)$ follows directly the expression of the consumers' surplus function given above. As for the proof of (ii), consider any two arbitrary links $i j$ and $k l$ where

\footnotetext{
${ }^{6}$ we delete $\eta_{i}$ in order to obtain the number of links formed in the network $g_{-i}$
} 
neither link belongs to the network. We have,

$$
\Delta C S(g+i j, k l)-\Delta C S(g, i j)=\frac{8 \gamma^{2}\left(n^{2}+4 n+5\right)}{\left(3+4 n+n^{2}\right)^{2}}>0 .
$$

It follows that the consumers' surplus function is convex in the total number of links formed.

Proof of Lemma 2. Suppose a network $g$ and firms $i, k, m \in N$ such that $i k, i m \notin g$ and $\eta_{m}(g) \geq \eta_{k}(g)$. The aggregate profit effect of link formation between $i$ and $k$ is given by $\Delta \Pi(g, i k)=\kappa^{\prime}\left(\alpha_{i}+\alpha_{k}-\frac{\tau^{\prime}}{n} \sum_{\ell \neq i, k} \alpha_{\ell}+\Lambda^{\prime}\right)-2 c$, where $\tau^{\prime}>0, \kappa^{\prime}>0$ and $\Lambda^{\prime}$ are constants independent of network structure and $\alpha_{i}$ and $\alpha_{k}$ are the values of the demand intercept parameters before link formation commences ${ }^{7}$.

$\tau^{\prime}$ has an upper bound less than 11 (at $n=2$, its value is 10.79) and is strictly decreasing in $n$. It has a lower bound of 2 and converges asymptotically to 2 . It is important to note that at $n=4, \tau^{\prime}=3.5$.

Hence, starting from an arbitrary network $g$ with $i k, i m \notin g$, if firms $i$ and $k$ form a link, the increase in aggregate profits can be written as $\frac{\Delta \Pi(g, i k)}{\kappa^{\prime}}=2 \gamma_{0}+$ $\gamma\left(\eta_{i}(g)+\eta_{k}(g)\right)-\frac{\tau^{\prime}}{n}\left((n-2) \gamma_{0}+\gamma \sum_{\ell \neq i, k, m} \eta_{l}(g)+\gamma \eta_{m}(g)\right)+\Lambda^{\prime}-\frac{2 c}{\kappa^{\prime}}$.

Suppose then that firms $i$ and $m$ form a link. The increase in aggregate profits can be written as $\frac{\Delta \Pi(g+i k, i m)}{\kappa^{\prime}}=\frac{\Delta \Pi(g, i k)}{\kappa^{\prime}}+\gamma\left(2+\left(1+\frac{\tau^{\prime}}{n}\right)\left(\eta_{m}(g)-\eta_{k}(g)-1\right)\right)$. Since $\eta_{m}(g) \geq \eta_{k}(g)$, we have $\frac{\Delta \Pi(g+i k, i m)}{\kappa^{\prime}} \geq \frac{\Delta \Pi(g, i k)}{\kappa^{\prime}}+\gamma\left(1-\frac{\tau^{\prime}}{n}\right)$.

We know that $\tau^{\prime}=3.5$ for $n=4$, hence $\frac{\tau^{\prime}}{n}<1$. Furthermore, we know that $\tau^{\prime}$ is strictly decreasing in $n$, hence $\frac{\tau^{\prime}}{n}$ is strictly decreasing in $n$. It follows that $\frac{\tau^{\prime}}{n}<1$ for all $n \geq 4$. As a result, we have $\frac{\Delta \Pi(g+i k, i m)}{\kappa^{\prime}}>\frac{\Delta \Pi(g, i k)}{\kappa^{\prime}}$.

\footnotetext{
${ }^{7}$ Detailed calculations are available in the working paper version of this paper: http: mpra.ub.uni-muenchen.de /28188/1/MPRA_paper _28188.pdf
} 
Proof of Lemma 3. Suppose a network $g$ and firms $i, k, m \in N$ such that $i k, i m \notin g$ and $\eta_{m}(g) \geq \eta_{k}(g)$. We know from Lemma 2 , that we have $\frac{\Delta \Pi(g+i k, i m)}{\kappa^{\prime}}>\frac{\Delta \Pi(g, i k)}{\kappa^{\prime}}$, with $\kappa^{\prime}$. Besides we know that the consumers' surplus function is convex (see Lemma $1)$, so we have $\frac{\Delta C S(g+i k, i m)}{\kappa^{\prime}}>\frac{\Delta C S(g, i k)}{\kappa^{\prime}}$.

Since the welfare function is defined as $W=\Pi+C S$ and the convexity property is preserved under addition of two convex functions, we then have $\frac{\Delta W(g+i k, i m)}{\kappa^{\prime}}>$ $\frac{\Delta W(g, i k)}{\kappa^{\prime}}$, completing the proof.

\section{References}

[1] Belhaj, M., Bervoets, S. and F. Deroïan, (2013) "Network Design under Local Complementarities", Working Papers halshs-00796487.

[2] Bulow, J.I., J.D. Geanakopolos and Paul D. Klemperer, (1985) "Multimarket Oligopoly: Strategic Substitutes and Complements", The Journal of Political Economy, Vol. 93, 488-511.

[3] Bernheim, B.D. and M.D. Whinston, (1990)"Multimarket Contact and Collusive Behavior", Rand Journal of Economics, Vol. 21, 1-26.

[4] Bloch, F., (1995) "Endogenous Structures of Association in Oligopolies", Rand Journal of Economics, Vol. 26, 537-556.

[5] Chakrabarti, S. and H. Haller, (2011) "An Analysis of Advertising Wars", Manchester School, Vol. 79, 100-124.

[6] Goyal, G. and S. Joshi, (2003) "Networks of Collaboration in Oligopoly", Games and Economic Behavior, Vol. 43, 57-85. 
[7] Goyal, G. and S. Joshi (2006) "Unequal Connections", International Journal of Game Theory, Vol. 34, 319-349.

[8] Hagedoorn, J. (2006) "Understanding the Cross-level Embeddedness of Inter-firm Partnership Formation", Academy of Management Review, Vol. 31, 670-680.

[9] Jackson, M. and A. Wolinsky, (1996) "A Strategic Model of Social and Economic Networks", Journal of Economic Theory, Vol. 71, 44-74.

[10] Spence, M., (1976) "Product Differentiation and Welfare", American Economic Review, Vol. 66, 407-414.

[11] Singh, N. and S. Vives, (1984) "Price and Quantity Competition in a Differentiated Duopoly", RAND Journal of Economics, Vol. 15, 546-554.

[12] Vives, X., (1999) Oligopoly Pricing: Old Ideas and New Tools, Cambridge, MA, and London: The MIT Press.

[13] Westbrock, B., (2010) "Natural Concentration in Industrial Research Collaboration", RAND Journal of Economics, Vol. 41, 351-371. 